\title{
Especies mexicanas de Curculionidae (Insecta: Coleoptera) asociadas con agaves (Asparagaceae: Agavoideae)
}

\author{
Mexican species of Curculionidae (Insecta: Coleoptera) associated to agaves (Asparagaceae: \\ Agavoideae)
}

\author{
Alberto Romo y Juan J. Morrone $e^{\bigotimes}$ \\ Museo de Zoología Alfonso L. Herrera, Facultad de Ciencias, Universidad Nacional Autónoma de México. Apartado postal 70-399, 04510 México D. \\ F., México. \\ 凶juanmorrone2001@yahoo.com.mx
}

\begin{abstract}
Resumen. Se estudiaron las especies de picudos o gorgojos (Coleoptera: Curculionidae) asociadas con agaves (Asparagaceae: Agavoideae) en México. Se registraron 5 especies asociadas con especies de Agave, Furcraea, Hesperoyucca, Polianthes y Yucca; de éstas, 4 pertenecen a la subfamilia Dryophthorinae (Scyphophorus acupunctatus, S. yuccae, Rhinostomus frontalis y Cactophagus spinolae) y 1 a la Baridinae (Peltophorus polymitus). Se presentan diagnosis, ilustraciones y una clave para la identificación de las 5 especies de curculiónidos asociados con agaves. Se establecen las siguientes sinonimias: Sphenophorus validus LeConte, 1858 = Cactophagus spinolae (Gyllenhal, 1838); y Zygops polymitus seminiveus LeConte, 1884, Z. p. leopardinus Desbrochers, 1891 y Z. p. suffusus Casey, $1892=$ Peltophorus polymitus Boheman, 1845.
\end{abstract}

Palabras clave: Agave, Curculionidae, Dryophthorinae, Rhynchophorini, Baridinae, Conoderini.

\begin{abstract}
Weevils (Coleoptera: Curculionidae) associated with agaves (Asparagaceae: Agavoideae) from Mexico are analyzed. Five species were recorded associated with species of Agave, Furcraea, Hesperoyucca, Polianthes, and Yucca. Four of these species belong to the subfamily Dryophthorinae (Scyphophorus acupunctatus, S. yuccae, Rhinostomus frontalis, and Cactophagus spinolae) and 1 belongs to the subfamily Baridinae (Peltophorus polymitus). Diagnoses, illustrations and a key are presented for identifying the 5 species of weevils found on agaves. The following synonymies are established: Sphenophorus validus LeConte, 1858 = Cactophagus spinolae (Gyllenhal, 1838); and Zygops polymitus seminiveus LeConte, 1884, Z. p. leopardinus Desbrochers, 1891, and Z. p. suffusus Casey, $1892=$ Peltophorus polymitus Boheman, 1845.
\end{abstract}

Key words. Agave, Curculionidae, Dryophthorinae, Rhynchophorini, Baridinae, Conoderini.

\section{Introducción}

La subfamilia Agavoideae (Asparagaceae) - conocida hasta hace unos años como Agavaceae- incluye plantas con o sin tallos aparentes, con hojas en roseta, suculentas, coriáceas o herbáceas, flores bisexuales y semillas de color negro (Gentry, 1982). Existen en casi todos los ambientes, incluyendo áreas tropicales y templadas (Rzedowski, 1981), Forman parte importante de los ecosistemas áridos y semiáridos de México, donde se presenta un alto grado de endemismo, Dentro de ellas, destacan los agaves (Agave), endémicos de América, cuya distribución abarca desde la región meridional de los Estados Unidos de América llegando hasta América Central. De un total de 131 especies, $120(91.6 \%)$ se distribuyen en México y el $8.4 \%$ restante se encuentra en los Estados Unidos

Recibido: 13 marzo 2012; aceptado: 22 junio 2012 de América, Guatemala, Belice, El Salvador, Nicaragua, Costa Rica, Ecuador y Colombia (Gentry, 1982). Las especies de este género se encuentran especialmente adaptadas a ambientes áridos y con baja precipitación anual (Cronquist, 1991), predominan en más de la mitad del territorio nacional (Rzedowski, 1981) y caracterizando el matorral desértico rosetófilo. La mayoría de las especies de agaves representan un recurso natural importante desde el punto de vista económico, ya que son materia prima para producir fibras textiles como el ixtle y el henequén o bebidas como el aguamiel, pulque, mezcal y tequila. Los picudos, como es el caso de Scyphophorus acupunctatus (Aquino-Bolaños et al., 2007, 2010; Servín et al., 2006; Solís et al., 2001), que atacan especies de agaves pueden llegar a causar daños graves a los cultivos, provocando pérdidas económicas cuantiosas

Nuestro objetivo es actualizar el conocimiento de las especies mexicanas de picudos asociadas con agaves. 
Si bien, existen varias publicaciones donde se registran estas especies, hasta el momento no hay diagnosis ni ilustraciones ni una clave para facilitar su identificación.

\section{Materiales y métodos}

Se examinaron ejemplares depositados en las siguientes colecciones: American Museum of Natural History, Nueva York, E.U.A. (AMNH); Colección Entomológica del Centro de Estudios en Zoología, Universidad de Guadalajara, Zapopan, Jalisco (CZUG); Colección Nacional de Insectos, Instituto de Biología, Universidad
Nacional Autónoma de México, México, D. F. (IBUNAM); Universidad Autónoma del Estado de Hidalgo, Pachuca, Hidalgo (UAEH); Museo de Zoología Alfonso L. Herrera, Facultad de Ciencias, Universidad Nacional Autónoma de México, México, D.F. (MZFC); Universidad Autónoma de Baja California, Baja California (UABC). Para este estudio, se consideraron aquellos ejemplares cuyos rótulos indicaban que habían sido coleccionados sobre agaves, además de analizar los registros previos en la literatura.

Cada especie tratada se acompaña de una breve diagnosis, sinonimia, distribución geográfica, plantas huésped, fotografías y mapas de distribución.

\section{Resultados}

Clave para la identificación de las Curculionidae asociadas con agaves

1. Longitud total menor a $10 \mathrm{~mm}$; tegumento con escamas blancas; antena con funículo de 7 antenómeros y clava compacta; mandíbulas sin dientes; ojos grandes, dorsalmente contiguos ocupando gran parte de la cabeza; canal prosternal para recibir el rostro en reposo; escutelo trapezoidal; coxas anteriores separadas por canal prosternal; tibias posteriores con diente bien desarrollado.. .Peltophorus polymitus 1'. Longitud total mayor a $10 \mathrm{~mm}$; tegumento sin escamas; antena con funículo de 6 antenómeros y clava truncada; mandíbulas con 2-3 dientes; ojos laterales; sin canal prosternal para recibir el rostro en reposo; escutelo triangular; coxas anteriores subcontiguas; tibias posteriores sin dientes......

2. Clava antenal con ápice alargado; pronoto subcircular; mandíbulas exodontas; escutelo pequeño; tarsómero 2 largo; tarsómero 3 fuertemente bilobulado; pigidio ligeramente expuesto. Rhinostomus frontalis 2'. Clava antenal con ápice levemente alargado, truncado o cóncavo; pronoto subrectangular o subtriangular; mandíbulas endodontas; escutelo grande; tarsómero 2 corto; tarsómero 3 bilobulado; pigidio claramente expuesto...... 3. Cuerpo robusto; élitros con franjas rojas-anaranjadas; clava antenal con ápice tomentoso cónico; escroba no extendida hacia superficie ventral; lóbulos dermales en tarsitos ausentes; tarsómero 3 con sedas abundantes en toda la superficie ventral. Cactophagus spinolae 3'. Cuerpo pequeño; élitros sin franjas; clava antenal con ápice trucado o cóncavo; escroba ligeramente extendida hacia superficie ventral; lóbulos dermales en tarsitos bien desarrollados; tarsómero 3 con sedas ventrales abundantes en parte apical.

4. Clava antenal con ápice cóncavo; antenómero funicular 2 de la misma longitud que el 3; rostro ligeramente corto y grueso; mandíbulas con 2 dientes; pronoto subrectangular, finamente punteado; escutelo pequeño; élitros con intervalos finamente punteados en 1-2 líneas; tarsómero 2 no excavado, con sedas apicales ventrales; pigidio corto.

Scyphophorus acupunctatus

4'. Clava antenal con ápice truncado ligeramente carenado; antenómero funicular 2 más largo que el 3; rostro ligeramente largo y delgado; mandíbulas con 3 dientes; pronoto subtriangular, profundamente punteado; escutelo grande; élitros con intervalos profundamente punteados en una sola línea; tarsómero 2 excavado, con sedas apicales laterales; pigidio largo

Scyphophorus yuccae

Familia Curculionidae

Subfamilia Dryophthorinae

La subfamilia Dryophthorinae posee 14 géneros y cerca de 1200 especies, de las cuales 127 especies y 14 géneros se distribuyen en México (Morrone y Cuevas, 2004). Se caracterizan por las antenas con clava truncada en el último antenómero funicular, brillante y desnudo; prementón no visible en vista ventral; uñas tarsales separadas ventral y dorsalmente por lóbulos dermales; edeago con surco o línea lateral; y apodemas edeagales en línea con el cuerpo edeagal en vista lateral (Thompson, 1992; Morrone, 2000; Morrone y Cuevas, 2004).

Tribu Rhynchophorini

Es el grupo más diverso de la subfamilia en el Nuevo Mundo, donde se encuentran representadas 4 subtribus (Rhynchophorina, Litosomina, Polytina y Sphenophorina) y 9 géneros (Anderson, 2002). Sphenophorina es la mejor representada en México, con 5 géneros: Cactophagus, Metamasius, Rhodobaenus, Scyphophorus y Sphenophorus. Se caracteriza por la forma de la articulación entre el fémur y 
la tibia, que consiste en un conjunto de pestañas; carecen del segundo segmento apical del diente de la tibia; y el escutelo es triangular, ensanchado cerca de la base (Anderson, 2002). En México existen 9 especies de Cactophagus, 17 de Metamasius, 51 de Rhodobaenus, 2 de Scyphophorus y 29 de Sphenophorus (Morrone y Cuevas, 2004).

Las especies de Scyphophorus se encuentran en especies de agave (maguey pulquero y henequenero), atacando los cultivos y provocando pérdidas económicas. El género posee sólo 2 especies. Scyphophorus acupunctatus, conocida como el gorgojo del sisal, es de importancia económica y se ha llevado accidentalmente junto con agaves a muchas partes del mundo (Hawai, Borneo, Java, Australia y África). Scyphophorus yuccae se alimenta de plantas de Yucca en el suroeste de los Estados Unidos y Baja California. Ambas especies son de color negro, aladas, carecen de escamas dorsales, son bastante robustas, por lo general de 10 a $19 \mathrm{~mm}$ de largo, con rostro y pronoto largo y dimorfismo sexual poco notorio. Como otros Rhynchophorini presentan el pigidio expuesto.
Scyphophorus acupunctatus Gyllenhal, 1838

Scyphophorus acupunctatus Gyllenhal, 1838: 857.

Scyphophorus anthracinus Gyllenhal, 1838: 858.

Scyphophorus interstitialis Gyllenhal, 1838: 858.

Rhynchophorus asperulus LeConte, 1857: 58.

Scyphophorus robustior Horn, 1873: 409.

Diagnosis. Longitud de 11 a $20 \mathrm{~mm}$. Color negro brillante, con fina puntuación en todo el cuerpo; mandíbulas bidentadas, endodontas; ojos laterales ovales poco separados ventralmente; antena geniculada con clava truncada, insertada en la base del rostro, funículo de 6 antenómeros, el 2 y 3 son de igual tamaño, clava con ápice tomentoso, cóncavo; rostro casi recto, largo, ligeramente ancho; pronoto subrectangular; escutelo pequeño, triangular, más largo que ancho; élitros con base emarginada; pigidio expuesto por detrás de los élitros, ligeramente corto; coxas anteriores subcontiguas, tarsómero 3 bilobulado con sedas ventrales abundantes en la parte apical, lóbulos dermales bien desarrollados, con un par de uñas simples (Figs. 1-3).
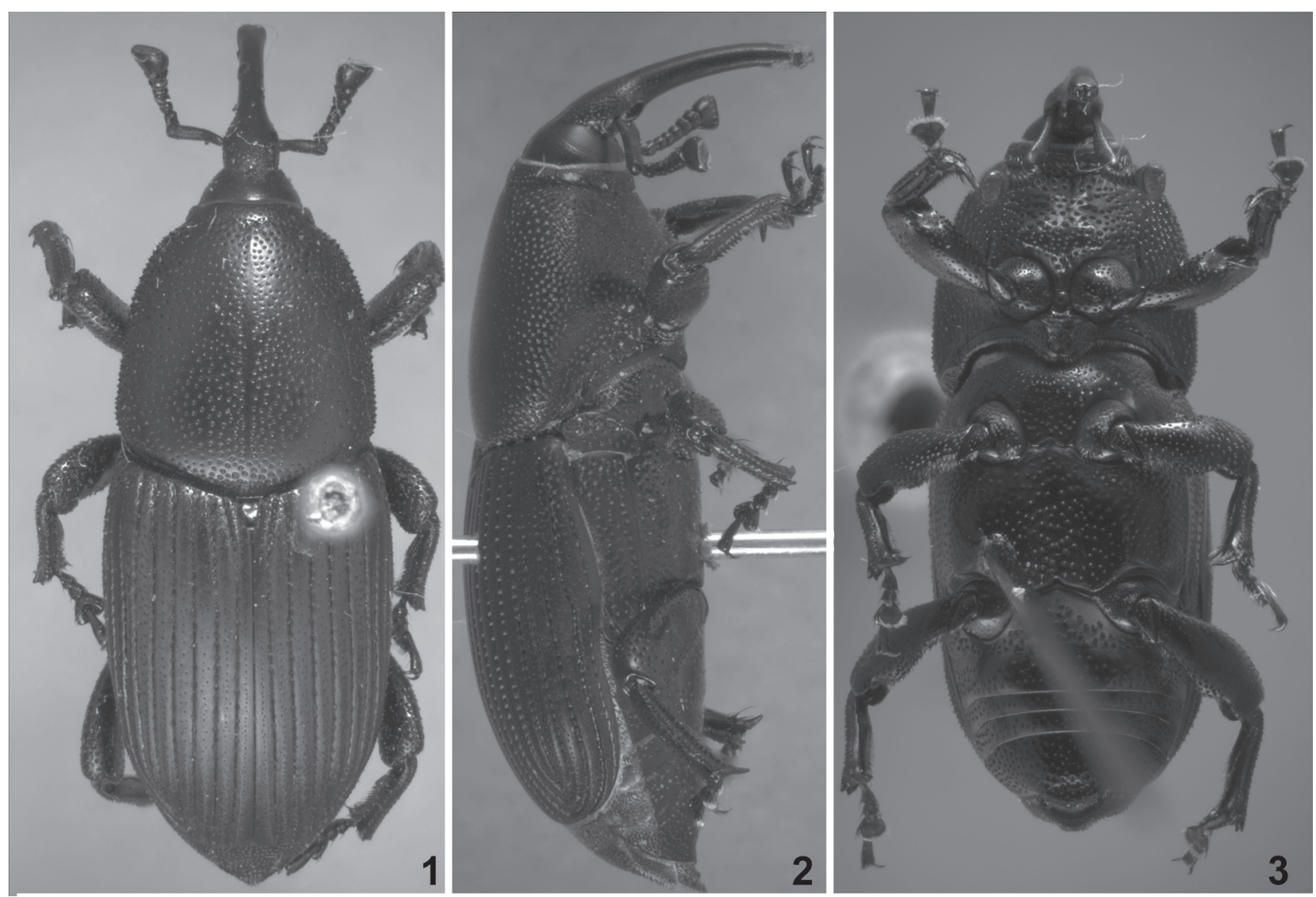

Figuras 1-3. Scyphophorus acupunctatus: 1, vista dorsal; 2, vista lateral; 3, vista ventral. 


\section{Resumen taxonómico}

Material examinado. México. Baja California Sur: Comitán, 30-XI-2010, 2 (UABC). Chiapas: Comitán, IV-1950, 4 (IBUNAM). Chihuahua: La Sauceda, 21-VII1947, 1 (AMNH). D.F.: Contreras, 10-VIII-1980, 2 (IBUNAM); Cuajimalpa, 6-II-1949, 1 (IBUNAM), 30-X1971, 2 (IBUNAM); La Venta, 16-IX-1959, 1 (IBUNAM); San Bartolo, 8-III-1947, 1 (IBUNAM); S Guadalupe, 4-VI-1960, 1 (IBUNAM). Estado de México: Chiconautla, 21-I-1961, 1 (IBUNAM); Santiago Tilapa, 30-VIII-1979, 1 (IBUNAM); Temascalpetec, 1931, 1 (IBUNAM); Tepotzotlán, 30-VI-1957, 1 (IBUNAM); Zumpango, 23-VIII-1947, 1 (IBUNAM). Hidalgo: Almoloya, 27-III1983, 6 (UNAM); Epazoyucan, 30-X-2002, 1 (UNAM), 30-X-2002, 2 (UAEH); Mineral de la Reforma, 15-VI2003, 1 (UAEH); Singuilucan, 22-X-2002, 2 (UAEH); Taxquillo, 5-II-1987, 1 (IBUNAM), 31-IX-2005, 4 (UAEH); Zempoala, 19-IX-2002, 5 (UAEH). Jalisco: Ajijic, 20-III-1948, 2 (IBUNAM). Michoacán: Pátzcuaro, 21-IX-2001, 3 (UAEH). Morelos: Amacozac, 21-VI1979, 1 (IBUNAM). Oaxaca: Tehuantepec, 2-V-1981, 1 (IBUNAM). San Luis Potosí: Huehuetlán, 29-XII-2003, 1 (IBUNAM). Tamaulipas: El Pachón, 13-V-1950, 1 (IBUNAM). Veracruz: Coatepec, 30-III-1990, 1 (UNAM); Las Vigas, 19-XI-1998, 1 (UAEH); Xalapa, 29-IV-1994, 1 (UNAM), 1 (AMNH). USA. Arizona: Portal, Cochise, 8-VI-1955, 1 (AMNH), 4-VIII-1959, 2 (AMNH).

Distribución. Sur de los Estados Unidos de América (Arizona, California, Colorado, Kansas, Nuevo México y Texas), prácticamente en todo México, Cuba, Jamaica, Haití, América Central y norte de América del Sur (Vaurie, 1971) (Fig. 4).

Plantas huésped. Agave amaniensis, A. americana, A. angustifolia, A. atrovirens, A. attenuata, A. ferdinandiregis, A. fourcroydes, A. karwinskii, A. lechuguilla, A. mexicana, A. potatorum, A. rigida, A. salmiana, A. shawii, A. sisalana, A. tequilana, Furcraea tuberosa, Polianthes tuberosa, Yucca aloifolia, Y. elephantipes, Y. glauca y Y. recurvifolia (Aquino-Bolaños et al., 2010; Maddison y Crosby, 2009; Pott, 1975; Solís et al., 2001; Vaurie, 1971; Woodruff y Pierce, 1973).

\section{Scyphophorus yuccae Horn, 1873}

Scyphophorus yuccae Horn, 1873: 410.

Diagnosis. Longitud del cuerpo de 15 a $20 \mathrm{~mm}$; color negro brillante, con puntuación profunda en todo el cuerpo; mandíbulas con 3 dientes, endodontas; ojos laterales ovales subcontiguos ventralmente; antena geniculada con clava truncada, insertada en la base del rostro, funículo de 6 antenómeros, el 2 es más largo que el 3, clava antenal con ápice tomentoso truncado, algo carenado; rostro levemente curvado, ligeramente delgado, largo; pronoto subtriangular; escutelo grande, triangular, tan largo como ancho; élitros con base casi recta; coxas anteriores subcontiguas; tarsómero 3 bilobulado, con sedas ventrales abundantes en la parte apical, lóbulos dermales bien desarrollados, con un par de uñas simples; pigidio expuesto por detrás de los élitros, ligeramente largo (Figs. 5-7).

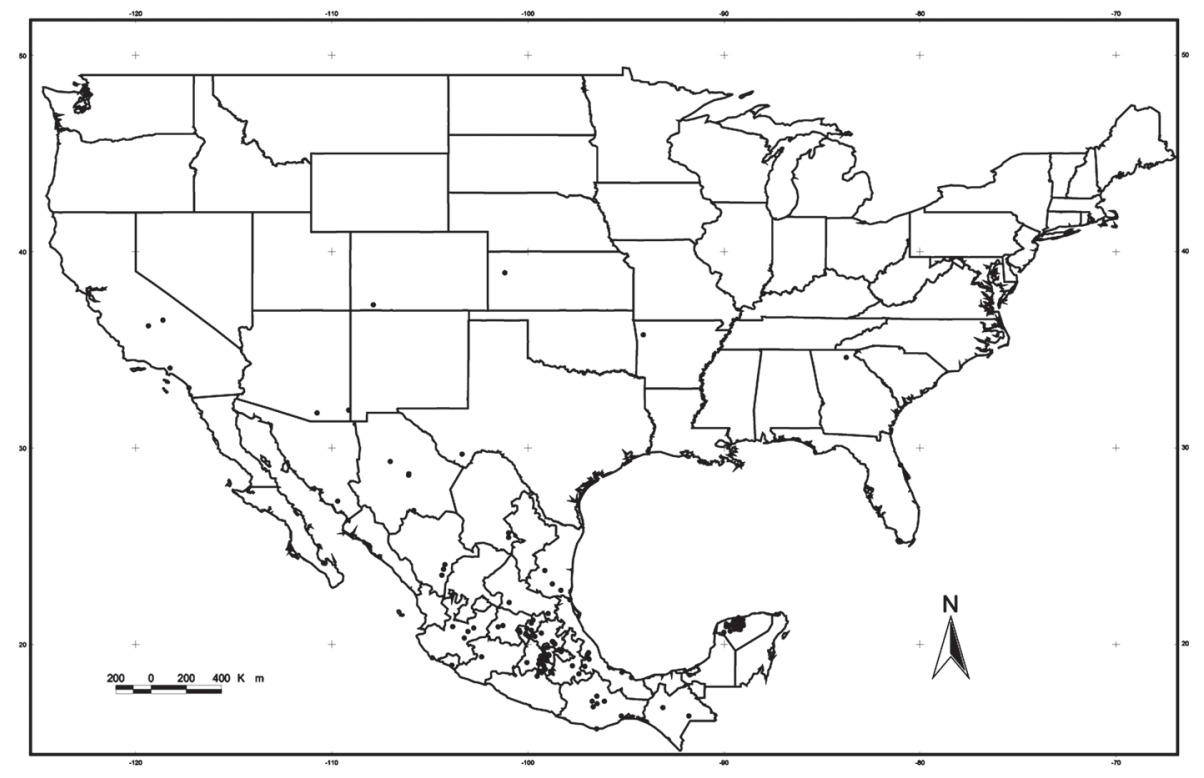

Figura 4. Mapa de distribución de Scyphophorus acupunctatus; registros de la literatura y ejemplares examinados. 

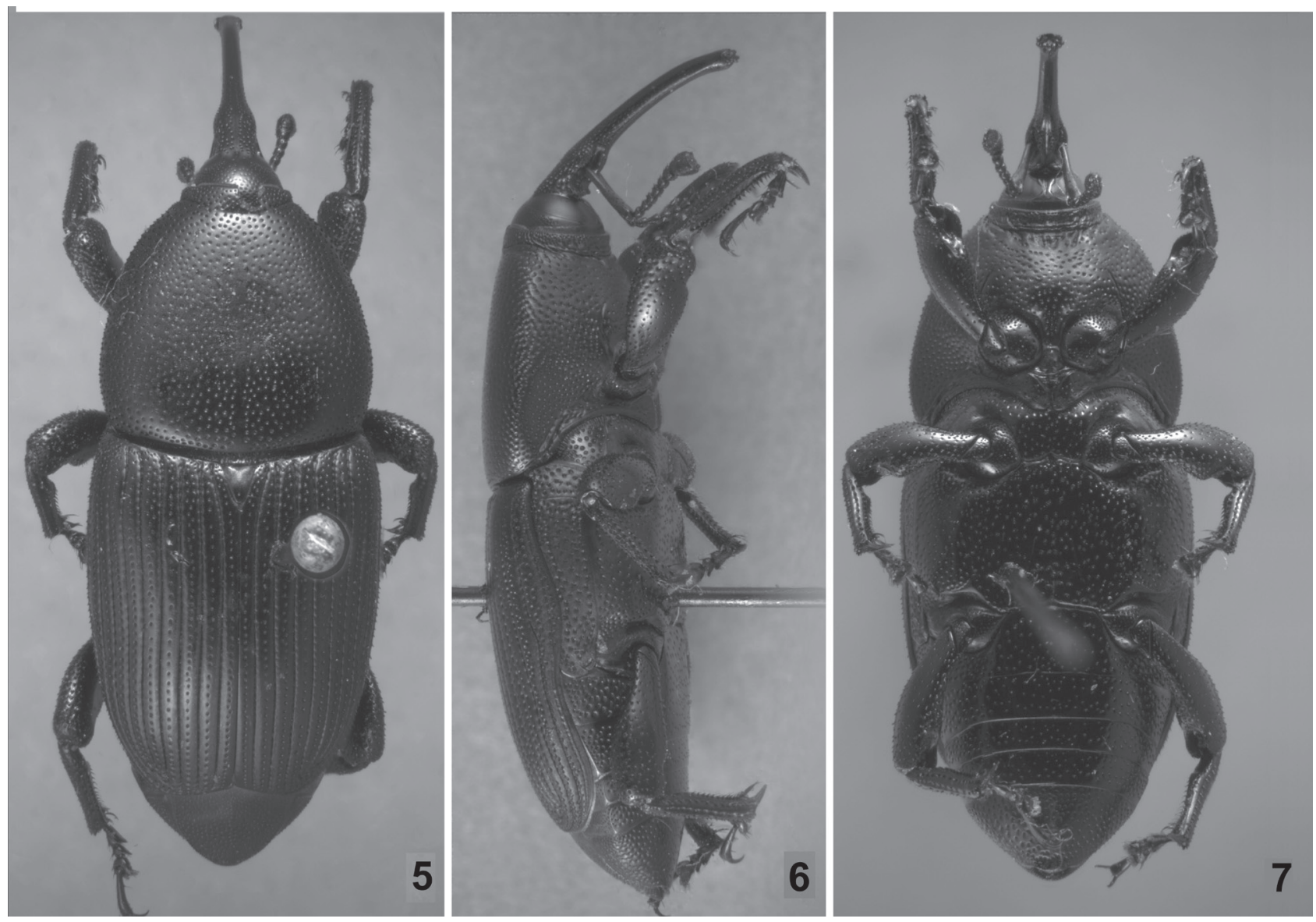

Figuras 5-7. Scyphophorus yuccae: 5, vista dorsal; 6, vista lateral; 7, vista ventral.

\section{Resumen taxonómico}

Material examinado. USA. California: Arroyo Seco, V-1959, 1 (AMNH); Cajon Pass, 14-V-1960, 1 (AMNH); Valle Vista, 30-IV-1961, 2 (AMNH).

Distribución. Sur de los Estados Unidos de América (California, Arizona y oeste de Texas) y en México al norte de Baja California (Huxman et al., 1997; Vaurie, 1971) (Fig. 8).

Plantas huésped. Agave amaniensis, Hesperoyucca whipplei y Yucca gloriosa (Maddison \& Crosby, 2009; Vaurie, 1971).

Cactophagus spinolae (Gyllenhal, 1838)

Sphenophorus spinolae Gyllenhal, 1838: 883.

Sphenophorus procerus LeConte, 1858: 80.

Sphenophorus validus LeConte, 1858: 80.

Cactophagus procerus; LeConte, 1876: 332.

Cactophagus validus; LeConte, 1876: 332.

Cactophagus obliquefasciatus Chevrolat, 1883: 580.

Cactophagus subnitens Casey, 1892: 685.

Cactophagus spinolae; Champion, 1910: 84.
Cactophagus rubronigrum Fischer, 1927: 217.

Metamasius procerus; Vaurie, 1966: 230.

Metamasius spinolae; Vaurie, 1966: 230.

Metamasius subnitens; Vaurie, 1966: 230.

Metamasius validus; Vaurie, 1966: 230.

Cactophagus spinolae validus; Kuschel, 1986: 374, syn. nov.

Diagnosis. Longitud del cuerpo de 15 a $25 \mathrm{~mm}$; color negro, cuerpo robusto; con 2 franjas tranversales de color rojo anaranjado en los élitros; mandíbulas con 3 dientes, endodontas; ojos laterales ovales separados ventralmente; antena geniculada con clava truncada, insertada en la base del rostro, funículo de 6 antenómeros, el 2 es más largo que el 3, clava antenal con ápice tomentoso cónico; rostro levemente curvado, grueso, largo; pronoto subtriangular; escutelo grande, triangular, más largo que ancho; élitros con base emarginada; coxas anteriores subcontiguas; tarsómero 3 bilobulado, con sedas abundantes en toda la superficie ventral, lóbulos dermales no desarrollados, con un par de uñas libres; pigidio expuesto por detrás de los élitros (Figs. 9-11). 
Nota nomenclatural: Consideramos que Sphenophorus validus LeConte, 1858, tratada recientemente como Cactophagus spinolae validus, no representa un taxón distinto de Cactophagus spinolae, por lo cual establecemos su sinonimia.

\section{Resumen taxonómico}

Material examinado. MÉXICO. D.F.: Milpa Alta, 2-VII1981, 1 (IBUNAM); Mixcoac, 15-VII-1952, 1 (IBUNAM); San Ángel, VI-1939, 1 (IBUNAM); San Jerónimo, 1974, 1 (IBUNAM); Xochimilco San Mateo Xalpa, 29-VI-1975,

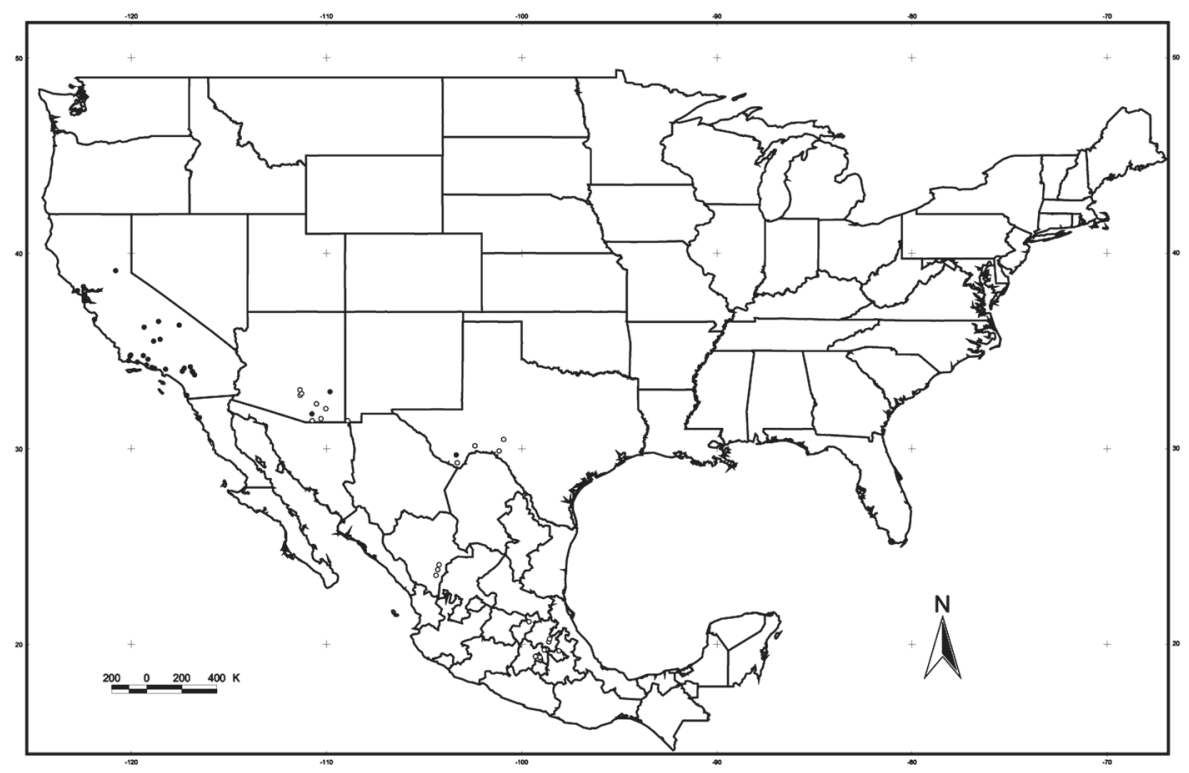

Figura 8. Mapa de distribución de Scyphophorus yuccae (•) y Peltophorus polymitus (०); registros de la literatura y ejemplares examinados.
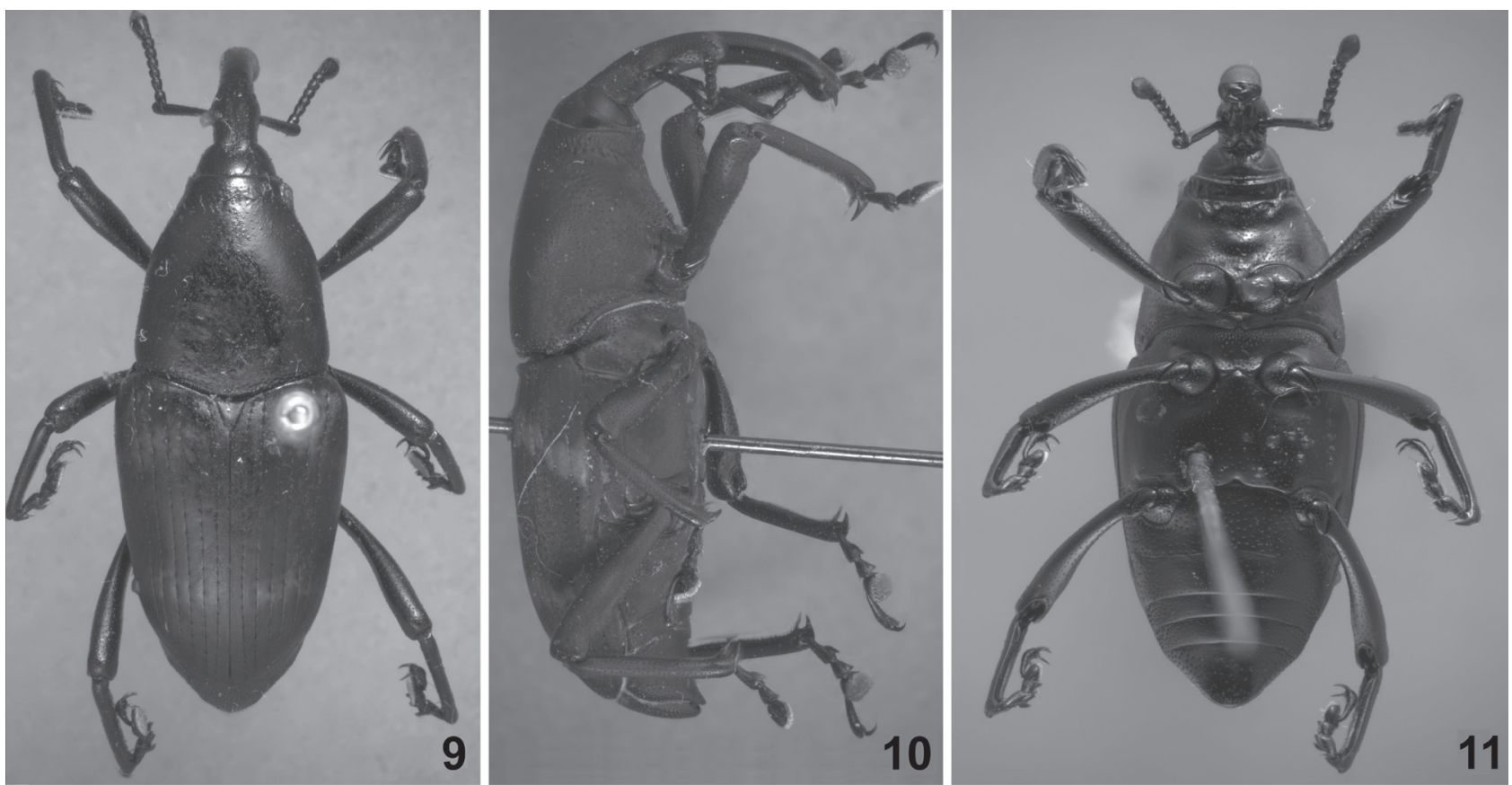

Figuras 9-11. Cactophagus spinolae: 9, vista dorsal; 10, vista lateral; 11 vista ventral. 
1 (IBUNAM). Durango: Palos Colorados, 5-VIII-1947, 1 (AMNH). Estado de México: Ameca-Meca, 27 -VIII1978, 1 (IBUNAM); Temascalpetec, 1931, 1 (IBUNAM); Tepotzotlán, 30-VI-1957, 1 (IBUNAM); Zoquiapan, 10-VIII-1958, 1 (IBUNAM). Guerrero: Chilpancingo, 28-V-1986, 2 (IBUNAM). Hidalgo: Zimapan El Tablón, 7-IV-2006, 1 (UAEH). Jalisco: Ajijic, 21-V-1947, 2 (IBUNAM), 7-IV-1950, 1 (IBUNAM); Zacoalco Lago Villa Corona, 13-VII-1953, 1 (AMNH); Zapopan, Agua Prieta, 900 m, 8-VI-1996, 6 (CZUG). Michoacán: Acámbaro, X-1916, 1 (IBUNAM); Maravatio, El Oro, km 62, 2-VII-1983, 1 (IBUNAM). Morelos: Cocoyoc, 6-VII1999, 1 (IBUNAM); Jonatepec, 19-IX-1984, 1 (IBUNAM); Laguna de Rodeo, 10-VII-1955, 1 (IBUNAM); Palmira, 22-VI-1947, 1 (IBUNAM); Tequesquitengo, 2-XI-1952, 1 (IBUNAM); Tlayacapan, 24-X-1948, 1 (IBUNAM). Oaxaca: Ojo de Agua, 12-VII-1973, 1 (IBUNAM); Huajuapan de León, IX-1937, 1 (IBUNAM). Zacatecas: Canutillo, 14-VIII-1947, 1 (AMNH).

Distribución. Sur de California y Arizona, y sur de Oaxaca hasta el norte de México (Vaurie, 1967) (Fig. 12).

Plantas huéspedes. Hylocereus spp., Opuntia spp. y Agave spp. (Ramírez-Delgadillo et al., 2011; Vaurie, 1967).

Tribu Orthognathini

Se caracteriza por presentar las coxas frontales contiguas. Comprende 2 subtribus: Orthognathina, con 3 géneros, y la subtribu monotípica Rhinostomina, que incluye el género Rhinostomus (Morrone y Cuevas, 2002,
2009). Rhinostomus es un taxón monofilético, definido por una combinación de los siguientes caracteres: mandíbulas exodontas, escapo antenal que pasa margen posterior del ojo, lóbulos postoculares ausentes, tibias por lo general dentadas, y tarsómero 3 bilobulado. Rhinostomus es pantropical y su distribución básicamente coincide con la de la familia de las palmas (Arecaceae). De sus 8 especies, 6 se encuentran en la región Neotropical y $R$. frontalis se extiende hasta a la región Neártica (Morrone y Cuevas, 2002).

Rhinostomus frontalis (LeConte, 1874)

Rhina frontalis LeConte, 1874: 70.

Yuccaborus frontalis; Casey, 1892: 688.

Yuccaborus sharpi Casey, 1892: 688.

Yuccaborus grossus Casey, 1892: 689.

Yuccaborus lentiginosus Casey, 1904: 324.

Yuccaborus frontalis sharpi; Vaurie, 1970: 49.

Rhinostomus frontalis; Morrone y Cuevas, 2002: 379.

Diagnosis. Longitud del cuerpo de 10 a $18 \mathrm{~mm}$, cuerpo alargado, color negro, con puntuación muy profunda en todo el cuerpo; mandíbulas con 3 dientes curvadas hacia afuera (exodontas); ojos laterales ovales casi unidos en la parte ventral; antena geniculada con clava truncada, insertada en la mitad del rostro, funículo de 6 antenómero, el 2 es más largo que el 3, clava antenal con ápice tomentoso alargado; rostro recto mediano y ancho; pronoto subcircular; escutelo muy pequeño, triangular; élitros con base casi recta; coxas anteriores subcontiguas; tarsómero 3 fuertemente bilobulado, con sedas ventrales abundantes

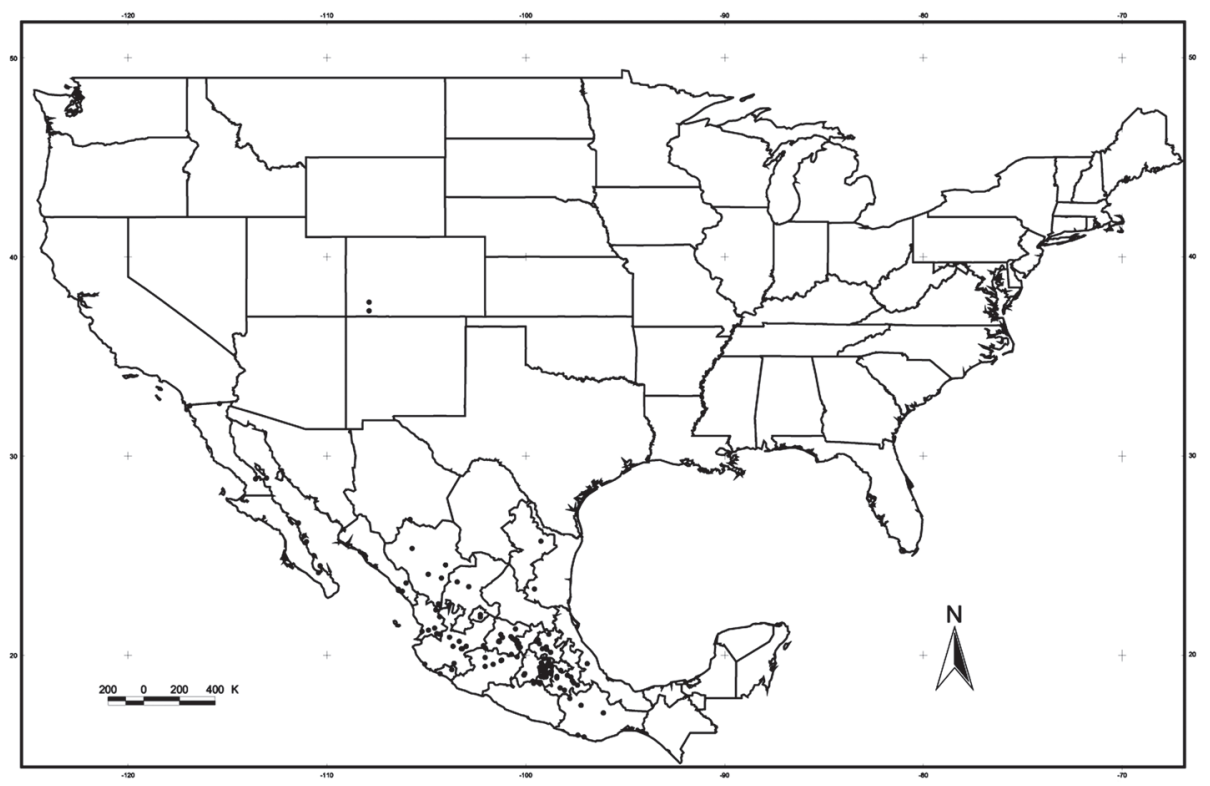

Figura 12. Mapa de distribución de Cactophagus spinolae; registros de la literatura y ejemplares examinados. 

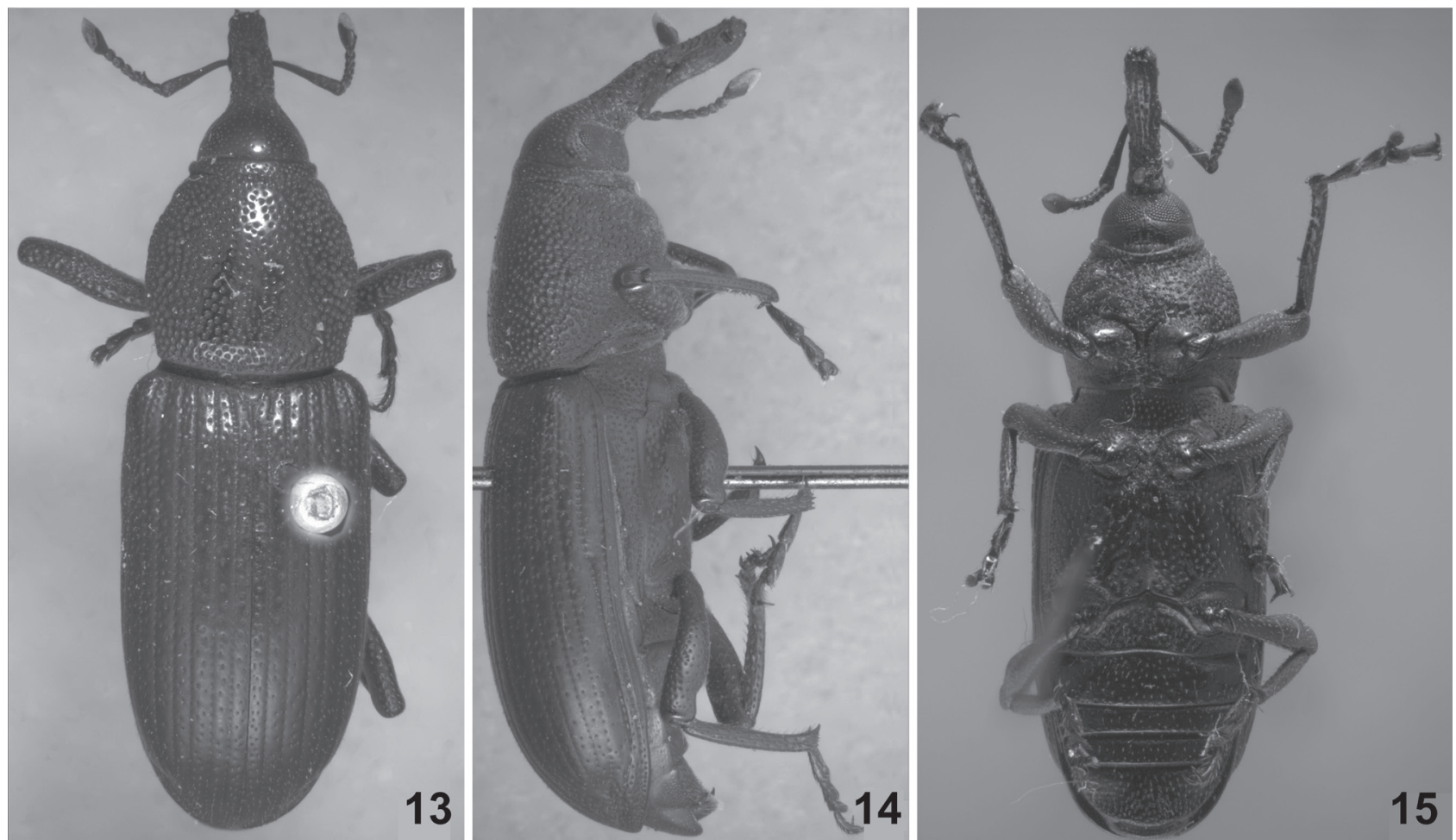

Figuras 13-15. Rhinostomus frontalis, 13, vista dorsal; 14, vista lateral; 15, vista ventral.

en forma de círculo en la parte ventral, lóbulos dermales bien desarrollados, con un par de uñas simples; pigidio levemente expuesto por detrás de los élitros (Figs. 13-15). Resumen taxonómico

Material examinado. México. Veracruz: Xalapa, 1 (AMNH). Zacatecas: Fresnillo, 15-VIII-1947, 2 (AMNH). USA. Arizona: Portal, Cochise, 3-VIII-1956, 1 (AMNH), 17-VII-1963, 1 (AMNH). California: Joshua Tree, 28-VIII11965, 1 (AMNH).

Distribución. Sur de los Estados Unidos de América (California, sur de Nevada y Texas) y México en Veracruz y Puebla (Vaurie, 1970) (Fig. 16).

Plantas huéspedes. Agave spp., Yucca valida y Yucca spp. (Vaurie, 1970)

\section{Subfamilia Baridinae}

La subfamilia Baridinae (sensu Zherikhin y Gratshev, 1995 ) incluye varios taxones tratados previamente como subfamilias independientes. La tribu Conoderini reconocida hasta hace poco tiempo como la subfamilia Zygopinae - se caracteriza por la presencia de un canal prosternal donde se aloja el rostro, grandes ojos que ocupan la mayor parte de la cabeza en algunos grupos y la ausencia de lóbulos postoculares (Kojima y Lyal, 2002).

Peltophorus polymitus Boheman, 1845
Peltophorus polymitus Boheman, 1845: 452.

Peltophorus leucomelas Lacordaire, 1866: 152 (nom. nud.).

Zygops polymitus seminiveus LeConte, 1884: 31, syn. nov.

Zygops polymitus leopardinus Desbrochers, 1891: 40, syn. nov.

Zygops polymitus suffusus Casey, 1892: 459, syn. nov. Peltophorus polymitis Hustache, 1934: 28 (lapsus).

Diagnosis. Longitud del cuerpo de 6 a $10 \mathrm{~mm}$, color negro, con escamas blancas en todo el cuerpo, presenta canal prosternal; mandíbulas sin dientes, endodontas; ojos redondos muy grandes, contiguos dorsalmente; antena geniculada con clava compacta, insertada en la base del rostro, funículo de 7 antenómeros, donde los antenómeros 1-3 son más largos que los antenómeros 4-7; rostro levemente comprimido dorsoventralmente, mediano; pronoto subtrapezoidal; escutelo trapezoidal; élitros con base fuertemente emarginada; coxas anteriores separadas por un canal prosternal; tarsómero 3 fuertemente bilobulado, con sedas abundantes en toda la superficie ventral, sin lóbulos dermales, con un par de uñas unidas en la base; pigidio expuesto por detrás de los élitros (Figs. 17-19).

Resumen taxonómico 


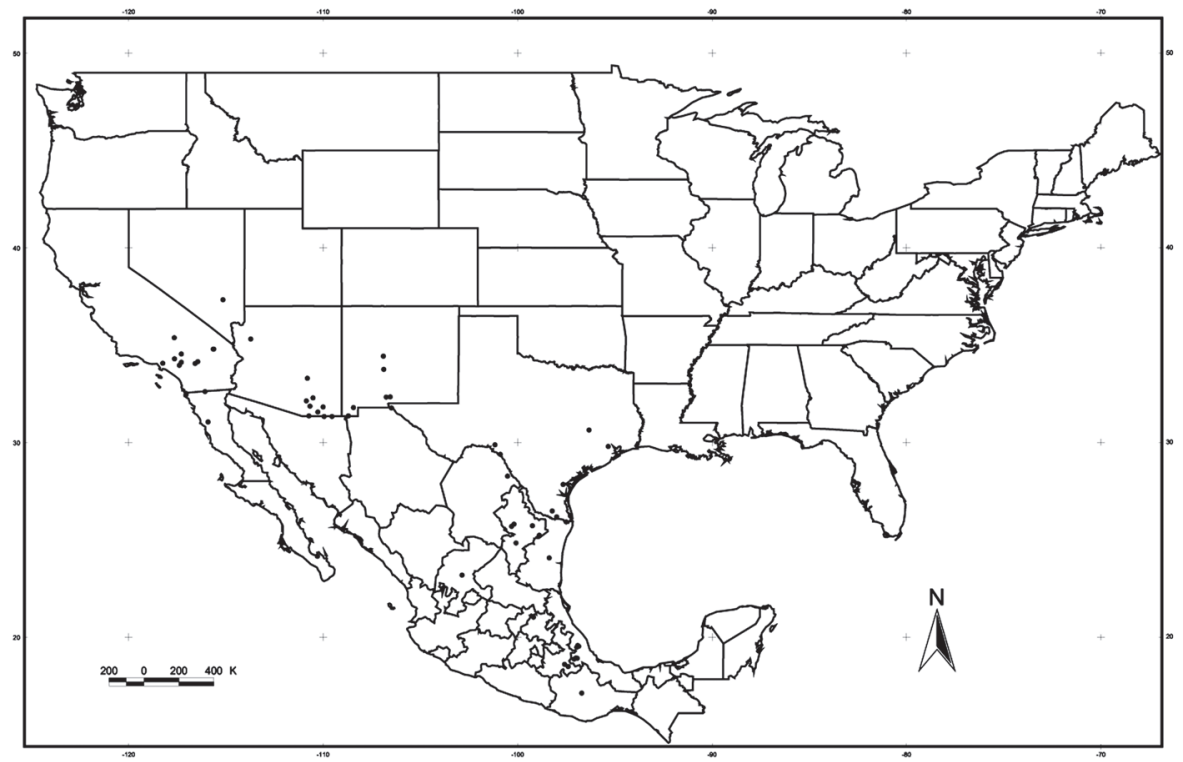

Figura 16. Mapa de distribución de Rhinostomus frontalis; registros de la literatura y ejemplares examinados.
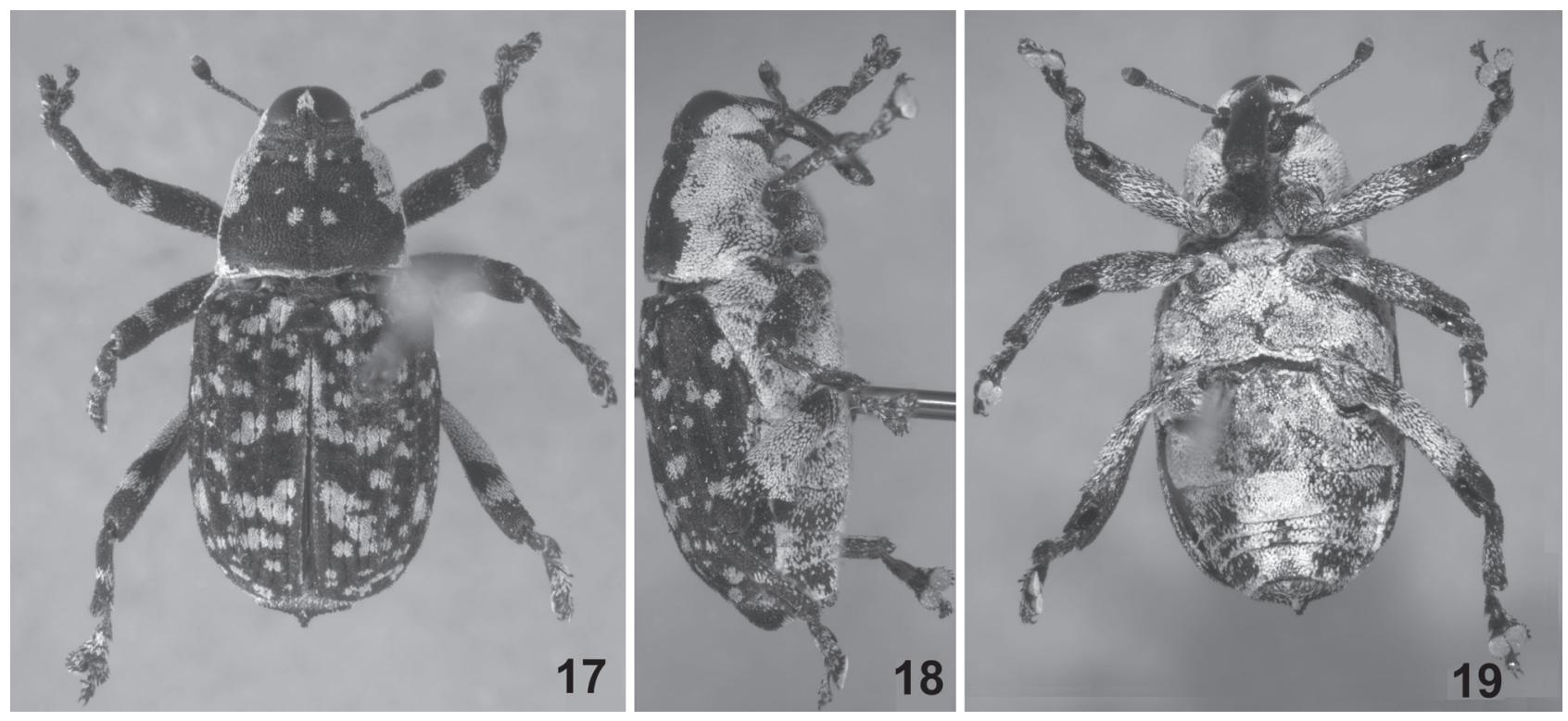

Figuras 17-19. Peltophorus polymitus: 13, vista dorsal; 14, vista lateral; 15, vista ventral.

Nota nomenclatural: Consideramos que Zygops polymitus seminiveus LeConte, 1884, Z. p. leopardinus Desbrochers, 1891 y Z. p. suffusus Casey, 1892: 459 no representan taxones distintos de Zygops polymitus, por lo cual establecemos su sinonimia.

Material examinado. México. D.F.: Cañada, 1 (IBUNAM); Cuajimalpa, 30-X-1971, 1, (IBUNAM), 17-VI-1972, 1 (IBUNAM); S Guadalupe, 4-VI-1960, 1
(IBUNAM). Hidalgo: Teotihuacán, 1 (IBUNAM), 30-X2002, 1 (AMNH), 30-X-2002, 1 (UNAM), 16-VII-2010, 1 (UAEH). Tlaxcala: Tlaxco, 10-X-2006, 1 (UNEH).

Distribución. Centro de México en D. F., Hidalgo y Tlaxcala (Fig. 8).

Plantas huéspedes. Agave durangensis, A. palmeri y Agave spp. (González-Castillo et al., 2011; Sleeper, 1963). 


\section{Comentarios taxonómicos}

En México existe una gran variedad de especies de agaves, de las cuales se cultivan el agave mezcalero (Agave angustifolia), el pulquero (A. atrovirens), el tequilero (A. tequilana) y el henequén ( $A$. fourcroydes). Diferentes enfermedades y plagas provocan daños a estas plantas, principalmente insectos barrenadores entre los que se encuentran los picudos o gorgojos (Coleoptera: Curculionidae), responsables de causar el mayor daño a la planta y provocar pérdidas económicas. Hasta el momento se han registrado 5 especies asociadas con agaves, pertenecientes a las subfamilias Dryophthorinae (Cactophagus spinolae, Scyphophorus acupunctatus, S. yuccae y Rhinostomus frontalis) y Baridinae (Peltophorus polymitus).

Scyphophorus acupunctatus es la principal plaga, ya que se encuentra asociada con 19 especies de agaves, varias de ellas de importancia económica: Agave americana, $A$. angustifolia, A. atrovirens, A. attenuata, A. ferdinandiregis, A. fourcroydes, A. karwinskii, A. lechuguilla, A. potatorum, A. salmiana, A. shawii, A. sisalana, A. tequilana. Furcraea tuberosa, Polianthes tuberosa, Yucca aloifolia, Y. elephantipes, Y. glauca y Y. recurvifolia. Su distribución es amplia, ya que se encuentra en gran parte del territorio mexicano y el sur de los Estados Unidos.

Scyphophorus yuccae ataca a Yucca gloriosa y Hesperoyucca whipplei pertenece al mismo género que $S$. acupunctatus y son especies muy similares, por lo que pueden llegar a ser confundidas. Su distribución se restringe al norte de México en Baja California y el sur de los Estados Unidos.

Rhinostomus frontalis ha sido registrada sobre Agave spp., Yucca valida y Yucca spp. Se distribuye en los estados de Baja California, Coahuila, Hidalgo, Nuevo León, Puebla, Oaxaca, Sonora, Tamaulipas, Veracruz y sur de los Estados Unidos.

Cactophagus spinolae ha sido asociada con Agave spp. (Vaurie, 1967). Si bien no se ha registrado una planta huésped específica, debería dársele especial atención, ya que tiene una amplia distribución en México y el sur de los Estados Unidos.

Peltophorus polymitus se registra para Agave palmeri y Agave spp. Su distribución se restringe al centro de México en el Distrito Federal, Hidalgo, Tlaxcala, Querétaro y el sur de los Estados Unidos.

Las diagnosis y la clave de identificación se basan en caracteres morfológicos externos de fácil observación con el uso de un microscopio estereoscópico, que deberían permitir la identificación correcta y sencilla de las especies asociadas con agaves, lo cual resulta importante desde el punto de vista agrícola. Creemos que este trabajo es relevante porque enfatiza la necesidad de actualizar el conocimiento taxonómico básico de estos insectos, imprescindible para llevar a cabo análisis sobre su ecología y factores que intervienen en su dinámica poblacional, y con ello tomar las medidas adecuadas para su manejo como plagas.

\section{Agradecimientos}

Agradecemos las valiosas sugerencias al manuscrito de José Luis Navarrete, Santiago Zaragoza Caballero y un revisor anónimo. También a los curadores de las distintas colecciones por el préstamo de los ejemplares a su cuidado. El primer autor agradece al Posgrado en Ciencias Biológicas de la Universidad Nacional Autónoma de México (UNAM) y el Consejo Nacional de Ciencia y Tecnología (CONACyT) por el apoyo económico para llevar a cabo sus estudios de Maestría.

\section{Literatura citada}

Anderson, R. S. 2002. The Dryophthoridae of Costa Rica and Panama: Checklist with keys, new synonymy and descriptions of new species of Cactophagus, Mesocordylus, Metamasius and Rhodobaenus (Coleoptera; Curculionoidea). Zootaxa 80:1-94.

Aquino-Bolaños, T., C. M. Iparraguirre y V. J. Ruiz. 2007. Scyphophorus acupunctatus (= interstitialis) Gyllenhal (Coleoptera: Curculionidae). Plaga del agave mezcalero: pérdidas y daños en Oaxaca, México. Agrícola 7:175-180.

Aquino-Bolaños, T., J. Ruiz-Vega y D. Martínez-Sánchez. 2010. Ecología y biología de Scyphophorus acupunctatus Gyllenhal (Coleoptera: Curculionidae), plaga del agave mezcalero en los Valles Centrales de Oaxaca. Naturaleza y Desarrollo 8:62-71.

Casey, T. L. 1892. Coleopterological notices II. Annals of the New York Academy of Sciences 6:359-712.

Casey, T. L. 1904. On some new Coleoptera, including five new genera. Canadian Entomologist 36:312-324.

Cronquist, A. 1991. Introducción a la botánica. Harper and Row, México, D. F. 848 p.

Gentry, H. S. 1982. Agaves of continental North America. The University of Arizona Press, Tucson, Arizona. $670 \mathrm{p}$.

González-Castillo, M. P., M. Q. Escalante y G. CastañoMeneses. 2011. Arthropods in natural communities in mescal agave (Agave durangensis Gentry) in an arid zone. American Journal of Applied Sciences 8:933-944.

Gyllenhal, L. 1838. In Schoenherr, C. J., Genera et species curculionidum, cum synonymia hujus familiae. Especies novae aut hactenus minus cognitae, descriptionibus a Dom. Leonardo Gyllenhal, C.H. Boheman, et entomologis aliis illustratae., 4, pt. 2, p. 601-1121. 
Horn, G. H. 1873. Contributions to a knowledge of the Curculionidae of the United States. Proccedings of the American Philosophical Society 13:407-447.

Hustache, A. 1934. Curculionidae: Zygopinae. In Coleopterorum Catalogus auspiciis et auxilio W. Junk, S. Schenkling (ed.). 136:1-96.

Huxman, T. E., K. A. Huxman y M. R. Stamer. 1997. Dispersal characteristics of the yucca weevil (Scyphophorus yuccae) in a flowering field of Yucca whipplei. Great Basin Naturalist 57:38-43.

Kojima, H. y C. H. Lyal. 2002. New Oriental and Australian Conoderinae, with taxonomic notes on the tribe Othippiini (Coleoptera: Curculionidae). Esakaia 42:161-174.

Lacordaire, T. 1866. Histoire naturelle des insectes. Genera des Coléopteres on exposé méthodique et critique de tous les genres proposés jusqu'ici dans cet ordre d'insectes, vol. 7. Roret, Paris. 620 p.

LeConte, J. L. 1857. Reports of explorations and surveys for a railroad route from the Mississippi River to the Paciffic Ocean. 9, 1: Report upon insects collected on the survey, 4. p. 1-72.

LeConte, J. L. 1858. Description of new species of Coleptera, chiefly collected by the United States and Mexican Boundary Commission, under Major W. H. Emory, U.S.A. Proceedings of the Academy of Natural Sciences of Philadelphia 10:59-89.

LeConte, J. L. 1874. The classification of the rhynchophorous Coleoptera. The American Naturalist 8:385-396, 452-470.

LeConte, J. L. 1884. Short studies of North American Coleoptera (No.2). Transactions of the American Entomological Society 12:1-32.

Maddison, P. A. y T. K. Crosby. 2009. Summary of plant-animal associations from "Maddison (1993) Pests and other fauna associated with plants, with botanical accounts of plants. Technical report. UNDP/FAO-SPEC Survey of Agricultural Pests and Diseases in the South Pacific, vol. 3. Auckland: Manaaki Whenua B Landcare Research". http://nzac. landcareresearch.co.nz/; última consulta: 15.I.2012.

Morrone, J. J. 2000. Mexican weevils (Coleoptera: Curculionoidea): A preliminary key to families and subfamilies. Acta Zoológica Mexicana 80:131-141.

Morrone, J. J. y P. Cuevas. 2002. Cladistics of the pantropical genus Rhinostomus (Coleoptera: Curculionoidea: Dryophthoridae), with nomenclatural notes. Journal of the New York Entomological Society 110:376-388.

Morrone, J. J. y P. Cuevas. 2004. Dryophthoridae (Coleoptera). In Biodiversidad, taxonomía y biogeografía de artrópodos de México: hacia una síntesis de su conocimiento, vol. IV, J. Llorente, J. J. Morrone, O. Yáñez e I. Vargas (eds.). Facultad de Ciencias, Universidad Nacional Autónoma de México, México, D. F. p. 705-712.

Morrone, J. J. y P. Cuevas. 2009. On the status of the tribes Orthognathini and Rhinostomini (Coleoptera: Curculionidae: Dryophthorinae). Zootaxa 2216:57-63.

O'Brien, C. W. y G. J. Wibmer. 1982. Annotated checklist of the weevils (Curculionidae sensu lato) of North America, Central America, and the West Indies (Coleoptera: Curculionoidea). Memoirs of the American Entomological Institute 34:1-382.

Pott, J. N. 1975. A yucca borer Scyphophorus acupunctatus in Florida. Proceedings of the Florida State Horticultural Society 88:414-416.

Ramírez-Delgadillo, J. J., E. Rodríguez-Leyva, M. LiveraMuñoz, A. Pedroza-Sandoval, N. Bautista-Martínez y C. Nava-Díaz. 2011. Primer informe de Cactophagus spinolae (Gyllenhal) (Coleoptera: Curculionidae) en tres especies de Hylocereus (Cactaceae) en Morelos, México. Acta Zoológica Mexicana (n. s.) 27:863-866.

Rzedowski, J. 1981. Vegetación de México. Limusa, México, D. F. 342 p.

Servín, R., A. Tejas, M. Montoya y M. Robert. 2006. Scyphophorus acupunctatus Gyllenhal (Coleoptera: Curculionidae) como potencial insecto-plaga de Yucca valida Brandegee en Baja California Sur, México. Folia Entomológica Mexicana 45:1-7.

Sleeper, E. L. 1963. A study of the Zygopinae (Coleoptera: Curculionidae) of America north of Mexico, I. Bulletin of the Southern California Academy of Sciences 62:209-220.

Solís, A. J. F., H. González, J. L. Leyva, A. Equihua, F. J. Flores y A. Martínez. 2001. Scyphophorus acupunctatus Gyllenhal, plaga del agave tequilero en Jalisco, México. Agrociencia 35:663-670.

Thompson, R. T. 1992. Observations on the morphology and classification of weevils (Coleoptera: Curculionoidea) with a key to the major groups. Journal of Natural History 26:835-891.

Vaurie, P. 1967. A revision of the Neotropical genus Metamasius (Coleoptera, Curculionidae, Rhynchophorinae). Bulletin of the American Museum of Natural History 136:177-268.

Vaurie, P. 1970. Weevils of the tribe Sipalini (Coleoptera, Curculionidae, Rhynchophorinae) Part 1. The genera Rhinostomus and Yuccaborus. American Museum Novitates 2419:1-57.

Vaurie P. 1971. Review of Scyphophorus (Curculionidae: Rhynchophorinae). Coleopterists Bulletin 25:1-8.

Woodruff, R. E. y W. H. Pierce. 1973. Scyphophorus acupunctatus, a weevil pest of Yucca and Agave in Florida (Coleoptera: Curculionidae). Entomology Circular No. 135.

Zherikhin, V. V. y V. G. Gratshev. 1995. A comparative study of the hind wing venation of the superfamily Curculionoidea, with phylogenetic implications. In Biology, phylogeny and classification of Coleoptera: papers celebrating the 80th birthday of Roy A. Crowson, J. Pakaluk y S. A. Ślipiński (eds.). Muzeum i Instytut Zoologii, Varsovia. p. 633-777. 